\title{
POTENCIAL INSETICIDA DE PLANTAS DA FAMÍLIA ANNONACEAE ${ }^{1}$
}

\author{
DIONES KRINSKI ${ }^{2,4}$, ANGÉLICA MASSAROLI ${ }^{3,4}$, MARILZA MACHADO $^{5,6}$
}

RESUMO- A utilização de plantas para o controle de insetos tem aumentado em todo o mundo. Isto ocorre porque os inseticidas vegetais apresentam moléculas biodegradáveis, são menos tóxicos a mamíferos e potencialmente adequados para utilização no controle de pragas. As plantas da família Annonaceae estão ganhando destaque como biopesticidas por serem naturalmente bioativas, apresentando atividade citotóxica, antitumoral, vermicida, antimicrobiana, imunossupressora, antiemética, inibidora do apetite e crescimento, antimalárica e também inseticida. A atividade inseticida das anonáceas deve-se à presença de acetogeninas, substâncias que atuam nas mitocôndrias, inibindo a NADH - ubiquinona oxidorredutase, causando a morte dos insetos. Nesta revisão, relatamos a utilização de anonáceas no controle de insetos, mostrando que até o momento, apenas 42 espécies de anonáceas possuem informações de atividade inseticida contra pouco mais de 60 espécies de insetos-praga. Estas informações mostram que muita pesquisa ainda é necessária, principalmente para conhecer a atividade inseticida de outras espécies de anonáceas, além de seus efeitos sobre insetos-praga ainda não estudados. Assim, teremos como alternativa para o desenvolvimento sustentável, a utilização de novos inseticidas vegetais, como os obtidos das diferentes espécies de anonáceas, os quais podem atuar como mais uma ferramenta para equilibrar os exageros da agricultura química ou convencional. Termos para indexação: plantas inseticidas; fitoinseticidas; controle alternativo.

\section{INSECTICIDAL POTENTIAL OF THE ANNONACEAE FAMILY PLANTS}

ABSTRACT- The use of plants compounds for the control of insects has increased worldwide. This occurs because the vegetal insecticides contains biodegradable compounds, nontoxic products and potentially suitable for use in pest control. Plants of the family Annonaceae are standing out as biopesticides because they are bioactive naturally in addition to presenting cytotoxic activity, antitumor, vermifuge, antimicrobial, immunosuppressive, anti-emetic, inhibiting appetite, antimalarial and also insecticide. The insecticidal activity of Annonaceae is due to the presence of acetogenins, substances that act on mitochondria inhibiting the NADH - ubiquinone oxidoreductase, causing the death of insects. In this review we report the use of Annonaceae in insect control, showing that so far, only 42 species of Annonaceae have information insecticidal activity against just over 60 species of insect pests. This information shows that much research is still needed, especially to get to know the insecticidal activity of other Annonaceae species, in addition to its effects on insect pests not yet studied. So we will have as an alternative to sustainable development, new vegetal insecticides such as those obtained from different Annonaceae species, which can act as an additional tool to balance the excesses of agriculture chemical or conventional.

Index terms: insecticide plants; phyto-insecticides; alternative control.

\footnotetext{
${ }^{1}$ (Trabalho 263-13). Recebido em: 05-08-2013. Aceito para publicação em: 17-01-2014. V Congresso Internacional \& Encontro Brasileiro sobre Annonaceae: do gene à exportação (19 a 23 de Agosto de 2013). Botucatu-SP.

${ }^{2}$ Doutorando em Zoologia. Mestre em Ecologia e Conservação da Biodiversidade. Email: diones.krinski@ufpr.br.

${ }^{3}$ Mestre em Zoologia. Bióloga. Email: angelicamassaroli@gmail.com

${ }^{4}$ Universidade Federal do Paraná (UFPR), Programa de Pós-Graduação em Zoologia, Departamento de Zoologia, Bairro Jardim das Américas, Caixa Postal 19020, 81531-980, Curitiba, PR, Brasil.

${ }^{5}$ Mestranda em Agronomia/Horticultura. Bióloga. Email: marilzabio@gmail.com

${ }^{6}$ Universidade do Estado de São Paulo, Faculdade de Ciências Agronômicas (UNESP-FCA). Programa de Pós-Graduação em Horticultura, Departamento de Horticultura. Fazenda Lageado, Bairro Portaria II, Rodovia Alcides Soares, Km 3. 18610-307, Botucatu, SP, Brasil.
} 


\section{Fitoinseticidas}

A utilização de plantas e dos seus derivados como inseticida é uma prática que vem sendo adotada pelo homem desde a idade antiga (VIEGAS-JÚNIOR, 2003). Roark (1947), há mais de 60 anos, relatou cerca de 1.200 espécies de plantas apresentando esse potencial, e hoje, mais de 2.000 espécies são conhecidas pelo seu potencial inseticida (SALAZAR, 1997). No início da primeira metade do século XX, essas substâncias eram muito utilizadas no controle de insetos, principalmente nos países tropicais (COSTA et al., 2004). Desta forma, é evidente que os produtos de origem vegetal ressurgem como alternativa promissora para o controle de insetos, especialmente após serem preteridos entre as décadas de 1930 e 1950 durante a Segunda Guerra Mundial, com a descoberta dos inseticidas sintéticos (organoclorados, organofosrados, carbamatos e piretroides), nesta época, sobretudo, pela utilização do DDT (D'AMATO et al. ,2002).

Os produtos à base de plantas, além de terem efeito inseticida comprovado, apresentam uma diversidade de compostos ativos, os quais agem sinergicamente, apresentando características atraentes, desalojantes ou repelentes, entre outras que podem ser empregados em sistemas de manejo integrado de pragas, como alternativas dirigidas para controle e monitoramento das populações de insetos (NAVARRO-SILVA et al., 2009). Isso se deve ao fato de as plantas, normalmente apresentarem em sua composição química, metabólitos primários e secundários. O metabolismo primário é importante para o desenvolvimento de toda a planta, estando presente em todas as células vegetais. Já, o metabolismo secundário é utilizado pelas plantas como forma de proteção aos microrganismos, insetos e outros artrópodes fitófagos (LUCAS et al., 2000). Por isso, os metabólitos secundários são candidatos naturais para o descobrimento de novos produtos que possam ser utilizados no controle de insetos.

O emprego de vegetais e de produtos preparados a partir de seus constituintes, tais como extratos e óleos essenciais, contra insetos em geral têm aumentado nos países industrializados, inclusive no Brasil, principalmente pelo fato de o País possuir uma flora rica e diversa. Ao observar os dados de literatura envolvendo publicações que utilizaram plantas inseticidas, percebe-se o quanto é vasta esta abordagem e pode-se observar a eficácia destes produtos como alternativa de controle contra insetos. E, além do mais, por estes apresentarem compostos biodegradáveis e produtos não tóxicos são, potencialmente, adequados para utilização no controle de pragas.

Portanto, diante do aumento dos trabalhos envolvendo extratos e óleos essenciais obtidos de diferentes espécies e partes de plantas, serão tratados, aqui, aqueles relacionados à família Annonaceae, visando a demonstrar a utilidade deste grupo vegetal sobre diversas espécies de insetos, enfatizando, assim, a importância desta nova abordagem científica no estudo do controle alternativo de insetos-praga.

\section{Anonáceas com potencial inseticida}

A família Annonaceae apresenta distribuição pantropical, sendo a América Central e a do Sul, a África e a Ásia os principais centros de diversidade desse grupo. Fora dos trópicos, o grupo é representado apenas por Asimina triloba (Linnaeus) Dunal, que ocorre desde o norte do Canadá até o sul da América do Norte (HEUSDEN, 1992). Na literatura, alguns autores mostram que existem distinções entre a quantidade de gêneros para a família, com relatos de 130 até, aproximadamente 200 gêneros, embora o número de espécies permaneça o mesmo, sendo representados por cerca de 2.500 espécies descritas (SAUQUET et al., 2003).

Seus representantes são plantas lenhosas, de porte arbóreo ou arbustivo. Entre as anonáceas de interesse comercial, encontram-se as espécies do gênero Annona, conhecidas popularmente como pinha, ata, cherimólia, marolo, fruta-de-conde, pinha-azeda, graviola e condessa (HEUSDEN, 1992). Este grupo de plantas também tem reconhecida importância farmacológica, atuando como matériaprima de cosméticos e perfumaria, uso na medicina natural, além de apresentar atividade antimicrobiana e inseticida devido à presença de acetogeninas, substâncias que, quando utilizadas contra insetos, atuam nas mitocôndrias, inibindo a NADH, o que causa a morte destes organismos (ZAFRA-POLO et al., 1996; LÜMMEN, 1998). As acetogeninas são substâncias naturalmente bioativas, demonstrando atividades citotóxica, antitumoral, inseticida, vermicida, antimicrobiana, imunossupressora, antiemética, inibidora do apetite e antimalárica; no entanto, aqui será abordada apenas a atividade inseticida desta família (CAVÉ et al., 1997; ALALI et al., 1999).

As acetogeninas constituem uma classe de produtos naturais promissora como protótipos de agentes inseticidas, sendo encontradas nas cascas de galhos e raízes, raízes e, principalmente, em sementes de plantas da família Annonaceae (BERMEJO et al., 2005; CASTILLO-SÁNCHEZ et al., 2010). A 
uvaricina foi a primeira acetogenina isolada a partir de Uvaria accuminata Oliv. em 1982, o que evidencia o quão recente são os estudos com estas substâncias (ALALI et al., 1999).

Infelizmente, a grande diversidade de espécies de Annonaceae conhecida cientificamente não reflete a quantidade de estudos destas plantas, visando ao controle de insetos-praga, como se pode observar no quadro 1. Isso se deve, provavelmente, à recente descoberta das propriedades biocidas das anonáceas contra insetos.

\section{Insetos-praga controlados por anonáceas}

Deve-se considerar um inseto como praga para fins de controle os organismos que se tornam capazes de provocar danos de importância econômica para a agricultura e/ou a saúde (GALLO et al., 2002). Segundo Imenes e Ide (2002), para a escolha do método mais adequado de controle destas pragas, é necessária a identificação do agente causador de dano, o conhecimento de sua biologia e comportamento, e a caracterização da área atingida e da intensidade da infestação. Além disso, deve-se restringir o uso de produtos químicos, sendo este utilizado apenas como última opção de controle (IMENES; IDE, 2002).

Apesar da eficiência dos inseticidas sintéticos, o uso indiscriminado destes produtos tem resultado na seleção de populações de insetos resistentes, problemas de ressurgimento das pragas alvos de controle, aparecimento de novas pragas (surtos de pragas secundárias), toxicidade para organismos não alvos e efeitos nocivos sobre o meio ambiente, colocando em risco a sustentabilidade dos ecossistemas, bem como a saúde da humanidade (HERNANDEZ; VENDRAMIN, 1996). Assim, a retomada dos estudos com fitoinseticidas devese principalmente à necessidade de obter novos compostos para utilização no controle de pragas, que sejam menos agressivos ao meio ambiente, sem problemas de resíduos nos alimentos, que evitem ou retardem o aparecimento de insetos resistentes e, ainda, que não apresentem ação sobre organismos benéficos.

Plantas da família Annonaceae apresentam em sua composição diversas substâncias com potencial inseticida, entre elas as acetogeninas são as mais importantes por apresentarem bioatividade contra diversas espécies de insetos (ALALI et al., 1999). Na literatura, são relatadas 42 espécies de Annonaceae com potencial inseticida, distribuídas em 14 gêneros (Annona, Artabotrys, Asimina, Cardiopetalum, Dennettia, Duguetia, Guatteria,
Monodora, Mkilua; Oxandra, Polyathia, Rollinia, Unonopsis e Xylopia) com destaque para as espécies Annona muricata Linnaeus (graviola) e Annona squamosa Linnaeus (fruta-do- conde, pinha) que atualmente são as espécies mais utilizadas para estudos de potencial inseticida (Quadro 1).

Até o momento, os estudos de avaliação da atividade inseticida com esta família foram realizados, sobretudo, com as principais ordens de insetos consideradas pragas, com destaque para Lepidóptera, Coleóptera, Hemíptera, Díptera e Blattodea. A quantidade de espécies de insetos estudadas sob a ação destas plantas é de 65, distribuídos entre as ordens Díptera (17 espécies); Lepidóptera (19 espécies), Coleóptera (16 espécies); Hemíptera (11 espécies) e Blattodea (2 espécies).

Quando fazemos uma busca na literatura dos trabalhos que relacionam a atividade fitoinseticida das espécies de anonáceas, podemos notar que grande parte dos estudos são realizados tanto com pragas urbanas (domésticas), representadas por espécies das ordens Díptera, Hemíptera e Blattodea, quanto com insetos de importância na agricultura, representadas pelas ordens Lepidóptera, Coleóptera e Hemíptera (Quadro 1).

\section{Anonáceas e o controle de insetos (pragas) urbanos}

Dentre as pragas urbanas, algumas espécies de insetos da ordem Díptera, principalmente dos gêneros Anopheles, Aedes e Culex, são importantes vetores de doenças como a malária, a febre amarela, e a dengue. Alles et al. (2009/2010) relatam que, no Brasil, o mosquito Culex quinquefasciatus Say 1823 (Culicidae) é responsável pela transmissão da Wichereria bancrofti (Cobbold, 1877) (Onchocercidae), o agente etiológico da filariose brancroftiana, e também um potencial vetor para o Vírus do Oeste do Nilo (VNO), que é originário da África e pode acometer o homem, causando desde febre, dores de cabeça, mialgias, encefalite severa e até meningite. Devido à importância deste mosquito, pesquisadores como Monzon et al. (1994), PérezPacheco et al. (2004), Daniel et al. (2011) e Allison et al. (2013) estudaram o efeito fitoinseticida de $A$. squamosa contra C. quinquefasciatus, e Kamaraj et al. (2011), contra C. tritaeniorhynchus Giles, 1901 (Culicidae), todos demonstrando o potencial desta espécie para o controle destes vetores. Outras espécies de Annonaceae também foram utilizadas, visando ao combate de C. quinquefasciatus como encontrado nos trabalhos de Murty et al. (1997) e de Kabir (2010), que estudaram o potencial 
inseticida, respectivamente, das espécies Polyalthia longifolia (Sonn.) e Artabotrys odoratissimus R. Br. Já Magadula et al. (2009) avaliaram citotoxicidade e as propriedades larvicidas de três espécies de Annonaceae (A. muricata, A. senegalensis e A. squamosa) contra outra espécie de Culex, o mosquito C. quinquefascintus Say (Culicidae), apresentando resultados promissores para as espécies A. senegalensis e $A$. squamosa.

Braga e Valle (2007) relatam que várias espécies de mosquitos do gênero Aedes, como Aedes aegypti (Linnaeus, 1762) (Culicidae), Aedes albopictus (Skuse, 1894) (Culicidae) e Aedes polynesiensis Marks, 1951 (Culicidae) são transmissores da dengue, entretanto $A$. aegypti é o vetor mais importante (DIAS; MORAES 2013). Embora essas três espécies de Aedes possam ser vetores da dengue, os estudos utilizando fitoinseticidas à base de anonáceas foram realizados somente contra duas delas: Aedes albopictus, que foi estudado apenas por Kempraj e Bhat (2011), que testaram a fitoatividade da espécie $A$ squamosa, e $A$. aegypti, que devido a sua importância como vetor da dengue possui várias pesquisas visando a seu controle a partir de diversas espécies de Annonaceae, tais como o trabalho de Aciole et al. (2011), que comparou a fitoatividade de três espécies de Guatteria (G. blepharophylla, G. friesiana e G. hispida), Feitosa et al. (2009), que avaliou o efeito larvicida de Rollinia leptopetala, além de diversos autores que pesquisaram a bioatividade de várias espécies de Annona (A. coriacea, A. crassiflora, A. foetida, A, glabra e $A$. muricata), visando ao controle do principal vetor da dengue, o $A$. aegypti (MORALES et al., 2004; BOBADILLA et al., 2005; OMENA et al., 2007; PARRA-HENAO et al., 2007a; GUARIDO, 2009; OLIVEIRA et al., 2010; MORAES et al., 2011; COSTA et al., 2012; DILL et al., 2012).

Outros estudos também avaliaram o efeito larvicida de anonáceas sobre quatro espécies de Anopheles, gênero dos principais mosquitos vetores da malária no Brasil. Morales et al. (2004) avaliaram o efeito de $A$. muricata contra Anopheles albimanus Wiedemann, 1820 (Culicidae), e Saxena et al. (1993), Daniel et al. (2011) e Kamaraj et al. (2011) que testaram, respectivamente, os efeitos de A. squamosa contra Anopheles stephensi Liston, 1901 (Culicidae), Anopheles gambiae Giles, 1926 (Culicidae) e Anopheles subpictus Grassi, 1899 (Culicidae). E além dos estudos com mosquitos, alguns pesquisadores avaliaram o efeito de algumas espécies de anonáceas contra duas espécies de baratas domésticas, ambos verificando a atividade fitoinseticida de A. muricata contra Blatella germanica Linnaeus, 1767 (Blattellidae) (ALALI et al., 1998) e Periplaneta americana (Linnaeus, 1758) (Blattidae) (ROBLEDO-REYES et al., 2008).

Mesmo com a crescente quantidade de pesquisas na busca por novos fitoinseticidas para controlar os insetos-praga urbanos, nota-se que mais pesquisas são necessárias visando, por exemplo, a isolar os componentes ativos das espécies de Annonaceae já estudadas, a fim de testá-los isoladamente e confirmar o potencial citotóxico, larvicida e inseticida dos compostos fitoquímicos presentes nestas plantas.

\section{Anonáceas e o controle de insetos na agricultura}

Um dos problemas associados ao uso de pesticidas sintéticos é que os insetos-praga desenvolvem rapidamente a resistência para estes compostos químicos e, por isso, novas pesquisas têm sido realizadas, principalmente na busca de compostos menos prejudiciais ao homem e ao ambiente. Não diferentemente, entre estas pesquisas, estão aquelas relacionadas com plantas da família Annonaceae, visando a verificar a atividade fitoinseticida destas plantas para o controle das principais pragas agrícolas. Estes estudos foram feitos principalmente contra pragas das ordens Lepidóptera, Coleóptera e Hemíptera (Quadro 1).

Os lepidópteros, em geral, alimentamse do limbo e das nervuras foliares, podendo ocasionar desfolhamento total em diversas culturas (LOURENÇÃO et al., 2010). Considerando isto, várias espécies de Annonaceae têm sido estudadas, visando ao controle dessas pragas. O primeiro estudo utilizando uma anonácea para o controle de uma lagarta foi com uma espécie de lagarta do gênero Spodoptera. Este grupo de lepidópteros é amplamente distribuído no mundo, e das 30 espécies descritas, metade é considerada praga de variadas culturas de importância econômica (POGUE, 2002). Mikolajczak et al. (1998) foram os primeiros a utilizar a anonácea $A$. triloba para o controle de Spodoptera eridania (Stoll, 1782) (Noctuidae). Após seus resultados, várias pesquisas com outras espécies difundiram-se, como a realização de bioensaios com A. cherimolia, A. cornifolia, A. dioica, A. montana, Oxandra cf. xylopioides, $R$. emarginata, $R$. silvatica, $U$. lindmanii, para o controle da lagarta-militar, Spodoptera frugiperda (Smith, 1797) (Noctuidae), um dos principais lepidópteros-praga, que se destacam por se alimentarem em mais de 80 espécies de plantas, incluindo o algodão, o milho e a soja 
(POGUE, 2002; SAITO et al., 2004; MAIRESSE, 2005; ÁLVAREZ et al., 2007; ROJANO et al., 2007; CAPINERA, 2008; BLESSING et al., 2010). Em outros estudos, Babu et al. (1998) e Pratibha et al. (2010) utilizaram, respectivamente, A. reticulata e A. squamosa para controlar lagartas Spodoptera litura (Fabricius, 1775) (Noctuidae), considerado um grave lepidóptero-praga, pois possui hospedeiros em diversas culturas economicamente importantes, tais como o algodão, o amendoim, a soja, o tomate, a batata-doce, entre outras (TONG et al., 2013).

Além desses estudos, $A$. atemoya foi estudada por Seffrin et al. (2010), visando ao controle da lagarta mede-palmo, Trichoplusia ni Hübner, 1802 (Noctuidae), uma praga polífaga, que tem como hospedeiros brássicas, tomate, pimentão, pepino, melancia, beterraba, alface, algodão, soja e plantas daninhas, como alface-selvagem, dente-de-leão e olerícolas em cultivo protegido (JOST; PITRE, 2002; JANMAAT; MYERS, 2003). Ainda podemos ampliar os exemplos de lepidópteros de importância econômica controlados por várias espécies de Annonaceae em experimentos laboratoriais, tais como Anagasta kuehniella (Zeller, 1879) (Pyralidae) e Corcyra cephalonica (Stainton, 1865) (Pyralidae) (COELHO et al., 2007), Tuta absoluta (Meyrick, 1917) (Gelechiidae) (SILVA et al., 2007), Anticarsia gemmatalis Hübner 1818 (Noctuidae) (FONTANA et al., 1998; SAITO et al., 2004; SCHLÜTER, 2006), Leucinodes orbonalis Guenée, 1854 (Pyralidae) (OWOSU, 2012), Plutella xylostella (Linnaeus 1758) (Plutellidae) (LAETAMIA; ISMAN, 2004; SCHLÜTER, 2006; TRINDADE et al., 2011), Pseudaletia sequax (Franclemont, 1951) (Noctuidae) (FONTANA et al., 1998), Crocidolomia pavonana (Fabricius, 1794) (Pyralidae) (DADANG;PRIJONO, 2009), Eurytides marcellus (Cramer 1777) (Papilionidae), Manduca sexta (Linnaeus, 1763) (Sphingidae) e Ostrinia nubilalis (Hübner, 1796) (Crambidae) (LEWIS et al., 1993), Ascia monuste orseis (Latreille, 1819) (Pieridae) (MAIRESSE, 2005) e Chrysodeixis includes (Walker, [1858]) (Noctuidae) (MASSAROLI et al., 2013) (Quadro 1).

Insetos desfolhadores da ordem Coleóptera também já foram controlados em pesquisas de laboratório, utilizando fitoinseticidas oriundos de algumas Annonaceae, mostrando que, mesmo insetos mais resistentes, como os besouros, podem ser controlados com inseticidas vegetais, com destaque para Epilachna vigintioctopunctata (Fabricius, 1775) (Coccinellidae) nos estudos com $A$. reticulata e $A$. senegalensis (KARUNARATNE ;ARUKWATTA, 2009), Leptinotarsa decemlineata, Say, 1824 (Chrysomelidae) com A. muricata (GUADAÑO et al., 2000), Oryctes rhinoceros (Linnaeus, 1758) (Dynastidae), utilizando A. senegalensis (SREELETHA; GEETHA, 2012), Acalymma vittatum (Fabricius, 1775) (Chrysomelidae), Diabrotica undecimpunctata Linnaeus 1758 (Chrysomelidae) e Epilachna varivestis Mulsant, 1850 (Coccinellidae), controlados com As. triloba (MIKOLAJCZAK et al., 1988).

Os hemípteros são outro grupo de insetospraga de importância econômica para muitas culturas, e várias espécies de anonáceas já foram testadas contra este grupo de insetos, como, por exemplo, em Tibraca limbativentris Stål, 1860 (Pentatomidae), praga importante em cultivos de arroz em todo o Brasil, controlada com extratos de sementes de Annona mucosa and A. crassiflora (KRINSKI; MASSAROLI, 2013), Oncopeltus fasciatus (Dallas, 1852) (Lygaeidae), controlados por A. cherimolia (ÁLVAREZ et al., 2008) e As. triloba (LEWIS et al., 1993); Dichelops melacanthus (Dallas, 1851) (Pentatomidae) com A. coriacea (SOUZA et al., 2007); Dipetalogaster maxima (Uhler 1894) (Reduviidae) com as espécies $A$. crassiflora e X. aromática (COELHO et al., 2009); Euschistus heros (Fabricius, 1798) (Pentatomidae) com A. crassiflora (OLIVEIRA; PEREIRA, 2009), A. mucosa (MAGALHÃES et al., 2011) e A. muricata (MIKAMI, 2011); Rhodnius neglectus Lent, 1954 (Reduviidae), utilizando as espécies $A$. crassiflora, A. mucosa (COSSOLIN et al., 2011), A. dioica (CARNEIRO et al., 2011; 2013) e $A$. reticulata (SCHMEDA;ARIAS, 1992); Rhodnius pallescens Barber, 1932 (Reduviidae) e Rhodnius prolixus Stål, 1859 (Reduviidae) usando $A$. muricata (PARRAHENAO et al., 2007b); Bemisia tabaci (Gennadius, 1889) (Aleyrodidae) e Dysdercus koenigii (Fabricius, 1775) (Pyrrhocoridae) com A. squamosa (REDDY et al., 1993; CRUZ-ESTRADA et al., 2013), e Aphis gossypii Glover, 1877 (Aphididae) e Dalbulus maidis (Delong \& Wolcott, 1923) (Cicadellidae) utilizando A. triloba (MIKOLAJCZAK et al., 1988).

Outrossim, pesquisas foram realizadas com insetos-praga que atacam os alimentos após a colheita, principalmente da ordem Coleóptera, que ocorrem em grãos armazenados, e várias espécies de anonáceas mostraram-se eficientes para o controle deste tipo de praga,apresentando um efeito fumigante em diversas espécies de besouros, tais como Callosobruchus maculatus (Fabricius, 1775) (Chrysomelidae), controlados com $A$. muricata (ADEOYE; EWETE, 2010) e $A$. senegalensis (AKU et al., 1998); Callasobruchus chinensis (Linnaeus, 1758) (Chrysomelidae), com A. senegalensis (KOTKAR et al., 2002); Sitophilus 
zeamais Motschulsky, 1885 (Curculionidae), controlados por $A$. muricata (HINCAPIÉ-LLANOS et al., 2008; ASMANIZAR ; IDRIS, 2012), por $A$. mucosa (RIBEIRO et al., 2013), e por $A$. senegalensis (NGAMO-TINKEU et al., 2004) e D. furfuracea (SILVA et al., 2013); Sitophilus oryzae Linnaeus, 1763) (Curculionidae), pelas espécies $A$. senegalensis (ASHOK et al., 2010), D. tripetala e $X$. aethiopica (UKEH et al., 2012); Zabrotes subfasciatus (Boheman, 1833) (Chrysomelidae), testados $\operatorname{com} A$. muricata (ARAÚJO, 2010); Tribolium castaneum (Herbst. 1797) (Tenebrionidae), com A. senegalensis (KHALEQUZZEMAN; SULTANA, 2006; ANITA et al., 2012); e por fim, Tenebrio molitor Linnaeus 1758 (Tenebrionidae), controlados com A. triloba (LEWIS et al., 1993).

Observando os traballhos citados nesta revisão, nota-se que várias espécies de Annonaceae podem ser utilizadas no controle de insetos-praga. No entanto, mais estudos são necessários, principalmente com pesquisas de semi campo e campo para verificar se os mesmos padrões encontrados em laboratório são mantidos sob condições reais, especialmente para utilização de pequenos produtores.

\section{Perspectivas futuras}

Considerando a diversidade de espécies de anonáceas conhecidas mundialmente, nota-se que apenas cerca de $2 \%$ das espécies desta família possuem informações acerca de seu potencial inseticida. Isso demonstra que, ainda, se tem muito para pesquisar com este grupo de plantas em relação ao conhecimento de suas propriedades inseticidas. Também devem-se ampliar os experimentos com outras espécies de insetos-praga ainda não estudadas.

Infelizmente, os fitoinseticidas constituem apenas $1 \%$ do mercado mundial de inseticidas (ISMAN, 1997). Isto pode ser um reflexo de algumas barreiras para a comercialização de inseticidas vegetais que impossibilitam sua maior participação no mercado de insumos agrícolas. Entre as limitações estão a escassez de recursos vegetais, a falta de padronização e de controle de qualidade das formulações, fatores estes que dificultam o registro desses fitoquímicos. No entanto, isso não tem impedido a busca de novos inseticidas vegetais, sendo este um novo campo de investigação, principalmente devido à grande variedade de substâncias presentes em toda a flora mundial, que se configura como um forte atrativo para novas pesquisas na área de controle de insetos (VIEGAS-JUNIOR, 2003).

Portanto, é evidente que a utilização de inseticidas obtidos a partir de plantas pode ser uma ferramenta vantajosa em relação aos inseticidas sintéticos, especialmente com a descoberta de novas substâncias, como as acetogeninas encontradas nas anonáceas (ALALI et al., 1999). Além disso, a obtenção de novos compostos bioativos pode ser importante para controlar novas pragas, como, por exemplo, contra a lagarta Helicoverpa armigera (Hübner, 1805) (Noctuidae), que até pouco tempo apresentava distribuição geográfica restrita à Europa, Ásia, África e Oceania, e, recentemente, foi registrada no Brasil (CZEPAK et al., 2013). Esta é uma espécie extremamente polífaga, e suas larvas já foram registradas em mais de 60 espécies de plantas cultivadas e silvestres, e em cerca de 67 famílias hospedeiras, incluindo Asteraceae, Fabeaceae, Malvaceae, Poaceae e Solanaceae (POGUE, 2004), podendo causar danos a diferentes culturas de importância econômica, como o algodão, leguminosas em geral, sorgo, milho, tomate, plantas ornamentais e frutíferas (MORAL-GARCIA, 2006).

Todavia, como os fitoinseticidas são provenientes de recursos renováveis e possuem uma mistura de vários compostos ativos que agem sinergicamente, estes fitoquímicos ainda devem ser mais bem estudados, visando a conhecer seu potencial também sobre outros organismos que não seriam o alvo do controle (BARRETO, 2005). Embora os primeiros estudos de seletividade realizados por Leatemia e Isman (2004) já mostram que insetos predadores das ordens Neuróptera e Hemíptera são pouco afetados pelos extratos de Annona.

Nesta revisão, observa-se que as anonáceas compõem uma rica fonte de pesquisa, e muitos dos exemplares estudados mostraram-se promissores. Todavia, para a inserção definitiva e segura de produtos vegetais no mercado, estudos sobre mecanismos de ação, fitotoxidade, real segurança a mamíferos e outros vertebrados, entre outros assuntos, ainda são necessários. Além disso, a maioria dos estudos que testaram a atividade inseticida das anonáceas, foi realizada em laboratório, portanto estudos em campo e semicampo devem ser conduzidos com o intuito de verificar se o mesmo padrão de resultados encontrados nos estudos em laboratório se mantém.

Devido aos grandes males causados pela utilização em larga escala de agrotóxicos e inseticidas sintéticos, existe a necessidade de desenvolver novas tecnologias a fim de minimizar esta utilização. Além disso, há crescente interesse por produtos orgânicos, livres de agrotóxicos, bem como a conscientização de produtores e consumidores levando-os a atitudes ecologicamente corretas. Todos estes aspectos impulsionam a busca por produtos alternativos que não agridam o ambiente. 
QUADRO 1- Espécies de Annonaceae e as partes utilizadas como fitoinseticida contra diferentes espécies de insetos.

\begin{tabular}{|c|c|c|c|}
\hline Espécie de Annocaceae & Parte* & Espécies de insetos (Ordem**) & Ref.*** \\
\hline Annona atemoya & $\mathrm{S}$ & Trichoplusia ni (Lep.) & 1 \\
\hline \multirow{2}{*}{ Annona cherimolia } & \multirow{2}{*}{$\mathrm{S}$} & Oncopeltus fasciatus (Hem.) & 2 \\
\hline & & Spodoptera frugiperda (Lep.) & 3 \\
\hline \multirow{9}{*}{ Annona coriacea } & \multirow{9}{*}{$\mathrm{S}$} & Aedes aegypti (Dip.) & 4 \\
\hline & & Aedes aegypti (Dip.) & 5 \\
\hline & & Aedes aegypti (Dip.) & 6 \\
\hline & & Aedes aegypti (Dip.) & 7 \\
\hline & & Anagasta kuehniella (Lep.) & 8 \\
\hline & & Corcyra cephalonica (Lep.) & 8 \\
\hline & & Dichelops melacanthus (Hem.) & 9 \\
\hline & & Euschistus heros (Hem.) & 10 \\
\hline & & Tuta absoluta (Lep.) & 11 \\
\hline \multirow{2}{*}{ Annona cornifolia } & \multirow{2}{*}{$\mathrm{F}$} & Anticarsia gemmatalis (Lep.) & 12 \\
\hline & & Spodoptera frugiperda (Lep.) & 12 \\
\hline \multirow{10}{*}{ Annona crassiflora } & $\mathrm{F} / \mathrm{G} / \mathrm{R}$ & Aedes aegypti (Dip.) & 13 \\
\hline & $\mathrm{R}$ & Aedes aegypti (Dip.) & 14 \\
\hline & \multirow[t]{2}{*}{$\mathrm{S}$} & Aedes aegypti (Dip.) & 15 \\
\hline & & Aedes aegypti (Dip.) & 7 \\
\hline & $\mathrm{R}$ & Dipetalogaster maxima (Hem.) & 15 \\
\hline & \multirow{5}{*}{$\mathrm{S}$} & Euschistus heros (Hem.) & 17 \\
\hline & & Euschistus heros (Hem.) & 10 \\
\hline & & Rhodnius neglectus (Hem.) & 18 \\
\hline & & Spodoptera frugiperda (Lep.) & 19 \\
\hline & & Tibraca limbativentris (Hem.) & 20 \\
\hline \multirow{4}{*}{ Annona dioica } & $\mathrm{S}$ & Aedes aegypti (Dip.) & 7 \\
\hline & $\mathrm{F} / \mathrm{G}$ & Anticarsia gemmatalis (Lep.) & 12 \\
\hline & $\mathrm{S}$ & Rhodnius neglectus (Hem.) & 21 \\
\hline & $\mathrm{F} / \mathrm{G}$ & Spodoptera frugiperda (Lep.) & 12 \\
\hline Annona diversifolia & $\mathrm{F} / \mathrm{G}$ & Anastrepha ludens (Dip.) & 22 \\
\hline Annona foetida & $\mathrm{S}$ & Aedes aegypti (Dip.) & 23 \\
\hline Annona glabra & $\mathrm{S}$ & Aedes aegypti (Dip.) & 14 \\
\hline Annona lutescens & $\mathrm{F} / \mathrm{G}$ & Anastrepha ludens (Dip.) & 21 \\
\hline \multirow{2}{*}{ Annona montana } & $\mathrm{F} / \mathrm{G}$ & Oncopeltus fasciatus (Hem.) & 24 \\
\hline & $\mathrm{F} / \mathrm{G}$ & Spodoptera frugiperda (Lep.) & 25 \\
\hline \multirow{6}{*}{ Annona тисоsа } & $\mathrm{S}$ & Aedes aegypti (Dip.) & 7 \\
\hline & $\mathrm{S}$ & Chrysodeixis includens (Lep.) & 26 \\
\hline & $\mathrm{S}$ & Euschistus heros (Hem.) & 27 \\
\hline & $\mathrm{G} / \mathrm{S}$ & Rhodnius neglectus (Hem.) & 18 \\
\hline & $\mathrm{F} / \mathrm{G} / \mathrm{S}$ & Sitophilus zeamais (Col.) & 28 \\
\hline & $\mathrm{S}$ & Tibraca limbativentris (Hem.) & 20 \\
\hline \multirow{21}{*}{ Annona muricata } & \multirow{3}{*}{$\mathrm{S}$} & Aedes aegypti (Dip.) & 29 \\
\hline & & Aedes aegypti (Dip.) & 30 \\
\hline & & Aedes aegypti (Dip.) & 31 \\
\hline & $\mathrm{F} / \mathrm{G}$ & Anastrepha ludens (Dip.) & 22 \\
\hline & \multirow{3}{*}{$\mathrm{S}$} & Anopheles albimanus (Dip.) & 29 \\
\hline & & Anticarsia gemmatalis (Lep.) & 32 \\
\hline & & Blatella germanica (Blat.) & 33 \\
\hline & $\mathrm{F}$ & Callosobruchus maculatus (Col.) & 34 \\
\hline & $\mathrm{F}$ & Culex quinquefascintus (Dip.) & 35 \\
\hline & \multirow{2}{*}{$\mathrm{S}$} & Euschistus heros (Hem.) & 36 \\
\hline & & Leptinotarsa decemlineata (Col.) & 37 \\
\hline & $\mathrm{F}$ & Leucinodes orbonalis (Lep.) & 38 \\
\hline & $\mathrm{S}$ & Periplaneta americana (Blat.) & 39 \\
\hline & F & Plutella xylostella (Lep.) & 40 \\
\hline & $\mathrm{S}$ & Plutella xylostella (Lep.) & 41 \\
\hline & & Pseudaletia sequax (Lep.) & 32 \\
\hline & & Rhodnius pallescens (Hem.) & 42 \\
\hline & $S$ & Rhodnius prolixus (Hem.) & 42 \\
\hline & $\mathrm{S}$ & Sitophilus zeamais (Col.) & 43 \\
\hline & & Sitophilus zeamais (Col.) & 44 \\
\hline & & Zabrotes subfasciatus (Col.) & 45 \\
\hline
\end{tabular}




\begin{tabular}{|c|c|c|c|}
\hline \multirow{4}{*}{ Annona reticulata } & $\mathrm{S}$ & Epilachna vigintioctopunctata (Col.) & 46 \\
\hline & $\mathrm{C}$ & Plutella xylostella (Lep.) & 47 \\
\hline & NI & Rhodnius neglectus (Hem.) & 48 \\
\hline & $\mathrm{S}$ & Spodoptera litura (Lep.) & 49 \\
\hline Annona salzmannii & $\mathrm{C}$ & Aedes aegypti (Dip.) & 50 \\
\hline \multirow{3}{*}{ Annona senegalensis } & $\mathrm{R}$ & Callosobruchus maculatus (Col.) & 51 \\
\hline & $\mathrm{F}$ & Culex quinquefascintus (Dip.) & 35 \\
\hline & NI & Sitophilus zeamais (Col.) & 52 \\
\hline \multirow{35}{*}{ Annona squamosa } & $\mathrm{F}$ & Aedes aegypti (Dip.) & 53 \\
\hline & $\mathrm{S}$ & Aedes albopictus (Dip.) & 54 \\
\hline & \multirow{2}{*}{$\mathrm{F}$} & Anopheles gambiae (Dip.) & 55 \\
\hline & & Anopheles gambiae (Dip.) & 56 \\
\hline & PI & Anopheles stephensi (Dip.) & 57 \\
\hline & \multirow[b]{2}{*}{$\mathrm{F}$} & Anopheles subpictus (Dip.) & 58 \\
\hline & & Bemisia tabaci (Hem.) & 59 \\
\hline & \multirow{4}{*}{$\mathrm{S}$} & Callasobruchus chinensis (Col.) & 60 \\
\hline & & Ceratitis capitata (Dip.) & 61 \\
\hline & & Crocidolomia pavonana (Lep.) & 62 \\
\hline & & Culex quinquefasciatus (Dip.) & 63 \\
\hline & \multirow{5}{*}{$\mathrm{F}$} & Culex quinquefasciatus (Dip.) & 55 \\
\hline & & Culex quinquefasciatus (Dip.) & 56 \\
\hline & & Culex quinquefasciatus (Dip.) & 53 \\
\hline & & Culex quinquefascintus (Dip.) & 35 \\
\hline & & Culex tritaeniorhynchus (Dip.) & 58 \\
\hline & \multirow{3}{*}{$\mathrm{S}$} & Drosophila melanogaster (Dip.) & 64 \\
\hline & & Dysdercus koenigii (Hem.) & 65 \\
\hline & & Epilachna vigintioctopunctata (Col.) & 46 \\
\hline & $\mathrm{G}$ & Musca domestica (Dip.) & 66 \\
\hline & $\mathrm{F}$ & Musca domestica (Dip.) & 67 \\
\hline & $\mathrm{S}$ & Musca nebulo (Dip.) & 68 \\
\hline & \multirow{2}{*}{$\mathrm{F}$} & Oryctes rhinoceros (Col.) & 69 \\
\hline & & Periplaneta americana (Blat.) & 70 \\
\hline & \multirow{3}{*}{$\mathrm{S}$} & Plutella xylostella (Lep.) & 71 \\
\hline & & Plutella xylostella (Lep.) & 62 \\
\hline & & Plutella xylostella (Lep.) & 41 \\
\hline & $\mathrm{R}$ & Plutella xylostella (Lep.) & 47 \\
\hline & \multirow{3}{*}{$\mathrm{S}$} & Sitophilus oryzae (Col.) & 72 \\
\hline & & Spodoptera litura (Lep.) & 49 \\
\hline & & Spodoptera litura (Lep.) & 73 \\
\hline & $\mathrm{F}$ & Tribolium castaneum (Col.) & 74 \\
\hline & \multirow{3}{*}{$\mathrm{S}$} & Tribolium castaneum (Col.) & 75 \\
\hline & & Trichoplusia ni (Lep.) & 1 \\
\hline & & Trogoderma granarium (Dip.) & 76 \\
\hline Artabotrys modestus & $\mathrm{C} / \mathrm{R}$ & Anopheles gambiae (Dip.) & 77 \\
\hline Artabotrys monteiroae & $\mathrm{C} / \mathrm{R}$ & Anopheles gambiae (Dip.) & 77 \\
\hline Artabotrys odoratissimus & $\mathrm{C}$ & Culex quinquefascintus (Dip.) & 78 \\
\hline \multirow{15}{*}{ Asimina triloba } & $\mathrm{R}$ & Acalymma vittatum (Col.) & 79 \\
\hline & $\mathrm{C}$ & Aedes aegypti (Dip.) & 79 \\
\hline & $\mathrm{C}$ & Aphis gossypii (Hem.) & 79 \\
\hline & $\mathrm{C}$ & Cerotoma trifurcata (Col.) & 80 \\
\hline & $\mathrm{C}$ & Colliphora vicina (Dip.) & 79 \\
\hline & $\mathrm{C}$ & Dalbulus maidis (Hem.) & 79 \\
\hline & $\mathrm{C}$ & Diabrotica undecimpunctata (Col.) & 79 \\
\hline & $\mathrm{C}$ & Epilachna varivestis (Col.) & 79 \\
\hline & NI & Eurytides marcellus (Lep.) & 81 \\
\hline & NI & Manduca sexta (Lep.) & 81 \\
\hline & NI & Oncopeltus fasciatus (Hem.) & 81 \\
\hline & NI & Ostrinia nubilalis (Lep.) & 81 \\
\hline & NI & Sarcophaga bullata (Dip.) & 81 \\
\hline & $\mathrm{C}$ & Spodoptera eridania (Lep.) & 79 \\
\hline & NI & Tenebrio molitor (Col.) & 81 \\
\hline \multirow{2}{*}{ Cardiopetalum calophyllum } & $\mathrm{F} / \mathrm{G} / \mathrm{R}$ & Aedes aegypti (Dip.) & 13 \\
\hline & $\mathrm{S}$ & Aedes aegypti (Dip.) & 7 \\
\hline
\end{tabular}




\begin{tabular}{|c|c|c|c|}
\hline \multirow{7}{*}{ Dennettia tripetala } & FR & Callosobruchus maculatus (Col.) & 82 \\
\hline & FR & Dermestes maculatus (Col.) & 83 \\
\hline & $\mathrm{S}$ & Dermestes maculatus (Col.) & 84 \\
\hline & NI & Ostrinia nubilalis (Lep.) & 85 \\
\hline & $\mathrm{S}$ & Necrobia rufipes (Col.) & 84 \\
\hline & FR & Sitophilus oryzae (Col.) & 86 \\
\hline & FR & Sitophilus zeamais (Col.) & 87 \\
\hline \multirow{2}{*}{ Duguetia furfuracea } & $\mathrm{F} / \mathrm{G} / \mathrm{R}$ & Aedes aegypti (Dip.) & 13 \\
\hline & $\mathrm{F}$ & Sitophilus zeamais (Col.) & 88 \\
\hline Guatteria blepharophylla & $\mathrm{F}$ & Aedes aegypti (Dip.) & 89 \\
\hline Guatteria friesiana & $\mathrm{F}$ & Aedes aegypti (Dip.) & 89 \\
\hline Guatteria hispida & $\mathrm{F}$ & Aedes aegypti (Dip.) & 89 \\
\hline Monodora myristica & $\mathrm{S}$ & Sitophilus zeamais (Col.) & 90 \\
\hline Mkilua fragrans & PA & Anopheles gambiae (Dip.) & 91 \\
\hline Oxandra cf xylopioides & $\mathrm{F}$ & Spodoptera frugiperda (Lep.) & 92 \\
\hline Polyalthia longifolia & $\mathrm{F}$ & Culex quinquefasciatus (Dip.) & 93 \\
\hline Rollinia emarginata & NI & Spodoptera frugiperda (Lep.) & 3 \\
\hline Rollinia leptopetala & $\mathrm{R}$ & Aedes aegypti (Dip.) & 94 \\
\hline Rollinia occidentalis & $\mathrm{S}$ & Spodoptera frugiperda (Lep.) & 95 \\
\hline Rollinia salicifolia & $\mathrm{F}$ & Anticarsia gemmatalis (Lep.) & 96 \\
\hline \multirow{3}{*}{ Rollinia silvatica } & $\mathrm{F}$ & Ascia monuste orseis (Lep.) & 97 \\
\hline & FR & Ostrinia nubilalis (Lep.) & 98 \\
\hline & $\mathrm{F}$ & Spodoptera frugiperda (Lep.) & 97 \\
\hline \multirow{2}{*}{ Unonopsis lindmanii } & $\mathrm{C}$ & Anticarsia gemmatalis (Lep.) & 12 \\
\hline & & Spodoptera frugiperda (Lep.) & 12 \\
\hline \multirow{3}{*}{ Xylopia aethiopica } & FR & Anopheles gambiae (Dip.) & 99 \\
\hline & $F K$ & Sitophilus oryzae (Col.) & 86 \\
\hline & NI & Ostrinia nubilalis (Lep.) & 100 \\
\hline \multirow{2}{*}{ Xylopia aromatica } & $\mathrm{F} / \mathrm{G} / \mathrm{R}$ & Aedes aegypti (Dip.) & 13 \\
\hline & $\mathrm{F}$ & Dipetalogaster maxima (Hem.) & 16 \\
\hline Xylopia caudata & $\mathrm{F}$ & Aedes aegypti (Dip.) & 101 \\
\hline Xylopia emarginata & $\mathrm{F} / \mathrm{G} / \mathrm{R}$ & Aedes aegypti (Dip.) & 13 \\
\hline Xylopia ferruginea & $\mathrm{F}$ & Aedes aegypti (Dip.) & 101 \\
\hline
\end{tabular}

$* \mathrm{C}=$ casca; $\mathrm{F}=$ folhas; $\mathrm{FR}=$ frutos; $\mathrm{G}=$ galhos; $\mathrm{R}=$ raiz; $\mathrm{S}=$ sementes; $\mathrm{PA}=$ parte aéreal $\mathrm{PI}=$ planta inteira; $\mathrm{NF}=$ não informado.

$* *$ Blat. $=$ Blattodea; Col. $=$ Coleoptera; Dip. $=$ Diptera; Hem. $=$ Hemiptera; Lep. $=$ Lepidoptera.

***1- Seffrin et al. (2010); 2- Álvarez-Colom et al. (2008); 3- Álvarez et al. (2007); 4- Costa et al. (2012); 5- Dill et al. (2012); 6- Moraes et al. (2011); 7- Costa et al. (2013) 8- Coelho et al. (2007); 9- Souza et al. (2007); 10- Silva et al. (2013) 11- Silva et al. (2007); 12- Saito et al. (2004); 13- Rodrigues et al. (2006); 14- Omena et al. (2007); 15- Oliveira et al. (2010); 16- Coelho et al. (2009); 17- Oliveira e Pereira (2009); 18- Cossolin et al. (2011); 19- Prates et al. (2000); 20- Krinski and Massaroli (2013); 21- Carneiro et al. (2011; 2013); 22- González-Esquinca et al. (2012); 23- Guarido (2009); 24- Álvarez et al. (2008); 25- Blessing et al. (2010); 26- Massaroli et al. (2013); 27- Magalhães et al. (2011); $28-$ Ribeiro et al. (2013); 29- Morales et al., (2004); 30- Bobadilla et al. (2005); 31- Parra-Henao et al. (2007a); 32- Fontana et al. (1998); 33- Alali et al., (1998); 34- Adeoye e Ewete (2010); 35- Magadula et al. (2009); 36- Mikami (2011); 37 Guadaño et al., (2000); 38- Owosu (2012); 39- Robledo-Reyes et al. (2008); 40- Trindade et al. (2011); 41- Sinchaisri et al. (1991); 42- Parra-Henao et al. (2007b); 43- Hincapié-Llanos et al. (2008); 44- Asmanizar e Idris (2012); 45- Araújo (2010); 46- Karunaratne e Arukwatta (2009); 47- Grainge et al. (1984); 48- Schmeda e Arias (1992); 49- Babu et al (1998); 50- Cruz (2011); 51- Aku et al. (1998); 52- Ngamo-Tinkeu et al. (2004); 53- Monzon et al. (1994); 54- Kempra e Bhat (2011); 55- Daniel et al. (2011); 56- Allisson et al. (2013); 57- Saxena et al. (1993); 58- Kamaraj et al. (2011); 59- Cruz-Estrada et al. (2013); 60- Kotkar et al. (2002); 61- Epino e Chang (1993); 62- Dadang e Prijono (2009); 63Pérez-Pacheco et al. (2004); 64- Kawazu et al. (1989); 65- Reddy et al. (1993); 66- Sharma et al. (2011); 67- Begum et al. (2010); 68- Qadri and Rao (1977); 69- Sreeletha e Geetha (2012); 70- Kesetyaningsih (2012); 71- Laetamia e Isman (2004); 72- Ashok et al. (2010); 73- Pratibha et al. (2010); 74- Anita et al. (2012); 75- Khalequzzeman e Sultana (2006) 76- Rao et al. (2005); 77- Nyandoro et al. (2013); 78- Kabir (2010); 79- Mikolajczak et al. (1988); 80- Ratnayake et al. (1993);81- Lewis et al. (1993); 82- Ukeh et al. (2011); 83- Akinwumi et al. (2007); 84- Okonkwo et al. (2001); 85Ewete et al. (1996); 86- Ukeh et al. (2012); 87- Umeotok et al. (2013); 88- Silva et al. (2013); 89- Aciole et al. (2011) 90- Ukeh et al. (2008); 91- Odalo et al. (2005); 92- Rojano et al. (2007); 93- Murty et al. (1997); 94- Feitosa et al. (2009); 95- Tolosa et al. (2012); 96- Schlüter (2006); 97- Mairesse (2005); 98- Mikolajczak et al. (1990); 99- Aina e al. (2009); 100- Ewete et al. (1996); 101- Zaridah et al. (2006). 


\section{REFERÊNCIAS}

ACIOLE, S. D. G.; PICCOLI, C. F.; DUQUE, J. E. L.; COSTA, E. V.; NAVARRO-SILVA, M. A.; MARQUES, F. A.; SALES MAIA, B. H. L. N.; PINHEIRO, M. L. B.; REBELO, M. T. Insecticidal activity of three species of Guatteria (Annonaceae) against Aedes aegypti (Diptera: Culicidae). Revista Colombiana de Entomología, Bogotá, v. 37, n.2, p.262-268, 2011.

ADEOYE, O. T.; EWETE, F. K. Potentials of Annona muricata Linnaeus (Annonaceae) as a botanical insecticide against Callosobruchus maculatus Fabricius (Coleoptera: Bruchidae). Journal of Agriculture, Forestry and the Social Sciences, Calabar, v. 8, n.2, p.147-151, 2010.

AINA, S. A.; BANJO, A. D.; LAWAL, O. A.; JONATHAN, K. Efficacy of some plant extracts on Anopheles gambiae mosquito larvae. Academic Journal of Entomology, Deira, v. 2, n.1, p.31-35, 2009.

AKINWUMI, F. O.; FASAKIN, E. A.; ADEDIRE, C. O. Toxic and repellence activities of four plant extracts to Dermestes maculatus Degeer on smoked african mud catfish, Clarias gariepinus Burchell. Journal of Entomology, New York, v. 4, n.2, p.149154, 2007.

AKU, A. A.; OGUNWOLU, E. O.; ATTAH, J. A. Annona senegalensis L. (Annonaceae): performance as a botanical insecticide for controlling cowpea seed bruchid, Callosobruchus maculatus (F.) (Coleoptera: Bruchidae) in Nigeria. Zeitschrift für Pflanzenkrankheiten und Pflanzenschutz, Stuttgart, v. 105, n.5, p.513-519, 1998.

ALALI, F. Q.; KAAKEH, W.; BENNETT, G.;MCLAUGHLIN, J. Annonaceous acetogenins as natural pesticides; potent toxicity against insecticidesusceptible and resistant german cockroaches (Dictyoptera: Blattellidae). Journal of Economic Entomology, Lanham, v. 91, n.3, p.641-649, 1998.

ALALI, F. Q.; LIU, X. X.; McLAUGHLIN, J. L. Annonaceous acetogenins: recent progress. Journal of Natural Products, Columbus, v. 62, n.3, p.50440, 1999.
ALLES, G. C.; HÜBNER, M.; FIUZA, L. M. Toxicologia de Bacillus thuringiensis às pragas urbanas e vetores. Biotecnologia Ciência \& Desenvolvimento, Brasília, v. 11, n.38, p.44-46, 2009/2010.

ALLISON, L. N.; DIKE, K, S.; OPARA, F. N.; EZIKE, M. N.; AMADI, A. N. Evaluation of larvicidal efficacy and phytochemical potential of some selected indigenous plant against Anopheles gambiense and Culex quinquefasciatus. Advances in Bioscience and Biotechnology, Irvine, v. 4, n.12, p.1128-1133, 2013.

ÁlVAREZ, O.; BARRACHINA, I.; AYALA, I., GONCALVEZ, M., MOYA, P., NESKE, A. y BARDÓN, A. Toxic effects annonaceous acetogenins on Oncopeltus fasciatus. Journal Pesticide Science, Tokyo, v. 81, n.2, p.85-89, 2008.

ÁLVAREZ, O., NESKE, A., POPICH, S.; BARDÓN, A. Toxic effects of annonaceous acetogenins from Annona cherimolia (Magnoliales: Annonaceae) on Spodoptera frugiperda (Lepidoptera: Noctuidae) Journal Pesticide Science, Tokyo, v. 80, n.1, p.63$67,2007 \mathrm{a}$.

ÁLVAREZ-COLOM, O.; POPICH, S.; BARDON, A. Bioactive constituents from Rollinia emarginata (Annonaceae). Natural Product Research: Formerly Natural Product Letters, London, v. 21, n. 3, p. 254-259, $2007 \mathrm{~b}$.

ANITA, S.; SUJATHA, P.; PRABHUDAS, P. Efficacy of pulverised leaves of Annona squamosa (L.), Moringa oleifera (Lam.) and Eucalyptus globulus (Labill.) against the stored grain pest, Tribolium castaneum (Herbst.). Recent Research in Science and Technology, Humnabad, v. 4, n.2, p.19-23, 2012.

ARAÚJO, A. M. N. Bioatividade de espécies vegetais em relação a Zabrotes subfasciatus (Boheman, 1983). (Coleoptera: Chrysomelidae: Bruchinae) em feijão (Phaseolus vulgaris L., 1753). 2010. 37f. Dissertação (Mestrado em Agronomia) - Ciências Agrárias, Universidade Federal de Alagoas, Maceió, 2010. 
ASHOK KUMAR, J.; REKHA, T.; DEVI, S. S.; KANNAN, M.; JASWANTH, A.; GOPAL, V. Insecticidal Activity of Ethanolic Extract of Leaves of Annona squamosa. Journal of Chemical and Pharmaceutical Research, Rajasthan, v.2, n.5, p.177-180, 2010.

ASMANIZAR, A. D.; IDRIS, A. B. Evaluation of Jatropha curcas and Annona muricata seed crude extracts against Sitophilus zeamais infesting stored rice. Journal of Entomology, New York, v. 9, n.1, p.13-22, 2012.

BABU, P. B. S.; RAO, J. M.; JOY, B. Effect of crude oils of Annona squamosa and Annona reticulata on feeding and development of Spodoptera litura (Fab.) larvae. Journal of Insect Science, Tucson, v. 10, p. 184-185, 1998.

BARRETO, C. F. Aedes aegypti: Resistencia aos inseticidas químicos e as novas alternativas de controle. Revista Eletrônica Faculdade Montes Belos, São Luis de Montes Belos, v. 1, n.2, p.62-73, 2005.

BEGUM, N.; SHARMA, B.; PANDEY, R. S. Evaluation of insecticidal efficacy of Calotropis procera and Annona squamosa ethanol extracts against Musca domestica. Journal of Biofertilizers \& Biopesticides, Sunnyvale, v. 1, n.1, p.1-6, 2010.

BERMEJO, A.; FIGADERE, B.; ZAFRA-POLO, M.C.; BARRACHINA, I.; ESTORNELL, E.; CORTES, D. Acetogenins from Annonaceae: recent progress in isolation, synthesis and mechanisms of action. Natural Product Reports, London, v. 22, n.2, p.269-303, 2005.

BLESSING, L. D. T.; ÁlVAREZ-COLOM, O.; POPICH, S.; NESKE, A.; BARDÓN, A. Antifeedant and toxic effects of acetogenins from Annona montana on Spodoptera frugiperda. Journal of Pest Science, Heidelberg, v. 83, n.3, p.307-310, 2010.

BOBADILLA, M.; ZAVALA, F.; SISNIEGAS, M.; ZAVALETA, G.; MOSTACERO, J.Y.; TARAMONA, L. Evaluación larvicida de suspensiones acuosas de Annona muricata Linnaeus (guanábana) sobre Aedes aegypti Linnaeus (Diptera, Culicidae). Revista Peruana de Biología, Lima, v. 12, n.1, p.15-152, 2005.
BRAGA, I. A.; VALLE, D. Aedes aegypti: inseticidas, mecanismos de ação e resistência. Epidemiologia e Serviços de Saúde, Brasília, v. 16, n.4, p.279-293, 2007.

CAPINERA, J. L. Encyclopedia of entomology. $2^{\text {nd }}$ ed. Dordrecht: Springer, 2008. v.1-4, 4346 p.

CARNEIRO, A. P.; PEREIRA, M. J. B.; GALBIATI, C. Biocide Activity of Annona coriacea extract on fourth and fifth instars nymphs and adults of the vector Rhodnius neglectus (Hemiptera, Reduviidae). Revista de Biologia Tropical, San José, v. 61, n.1, p.419-427, 2013.

CARNEIRO, A. P.; PEREIRA, M. J. B.; GALBIATI, C. Efeito biocida do extrato de Annona coriacea Mart 1841 sobre ovos e ninfas recém-eclodidas do vetor da doença de Chagas Rhodnius neglectus Lent 1954 (Hemiptera, Reduviidae). Neotropical Biology And Conservation, São Leopoldo, v.6, n.2, p.131136, 2011.

CASTILLO-SÁNCHEZ, L. H. C.; JIMÉNEZOSORNIO, J. J.; DELGADO-HERRERA, M. A. Secondary metabolites of the Annonaceae, Solanaceae and Meliaceae families used as biological control of insects. Tropical and Subtropical Agroecosystems, Yucatán, v. 12, n.3, p.445-462, 2010.

CAVÉ, A.; FIGADÈrE, A.; LAURENS, A.; CORTES, D. Acetogenins from Annonaceae. In: HERZ, W.; KIRBY, G. W.; MOORE, R. E.; STEGLISH, W.; TAMM, C. (Ed.). Progress in the Chemistry of Organic Natural Products. New York: Springer Verlag, 1997. p. 281-287.

COELHO, A. A. M.; PAULA, J. E.; ESPÍNDOLA, L. S. Efeito de extratos de plantas do Cerrado em Dipetalogaster máxima (Uhler) (Hemiptera, Reduviidae). Revista Brasileira de Entomologia, Curitiba, v.5 3, n.3, p.444-451, 2009.

COELHO, M. B.; MARANGONI, S.; MACEDO, M. L. Insecticidal action of Annona coriacea lectin against the flour moth Anagasta kuehniella and the rice moth Corcyra cephalonica (Lepidoptera: Pyralidae). Comparative Biochemistry and Physiology - Part C: Toxicology \& Pharmacology, New York, v. 146, n.3, p.406-14, 2007. 
COSSOLIN, J.F.S.; CARNEIRO, A. P.; PEREIRA, M. J. B.; SOUZA, P. T.; DALL'OGLIO, E. L. Atividade inseticida de extratos de Annona mucosa e $A$. crassiflora sobre Rhodnius neglectus (Hemiptera: Reduviidae). In: CICLO DE ESTUDOS EM BIOLOGIA DE TANGARÁ DA SERRA, CICLO NACIONAL DE ESTUDOS DE BIOLOGIA, 2., 2011, Tangará da Serra. Anais... 2011.

COSTA, E. L. N.; SILVA, R. F. P.; FIUZA, L. M. Efeitos, aplicações e limitações de extratos de plantas inseticidas. Acta Biologica Leopoldensia. São Leopoldo, v. 26, n.2, p.173-185, 2004.

COSTA, M. S.; PINHEIRO, D. O.; SERRÃO, J. E.; PEREIRA, M. J. B. Morphological changes in the midgut of Aedes aegypti L. (Diptera: Culicidae) larvae following exposure to an Annona coriacea (Magnoliales: Annonaceae) extract. Neotropical Entomology, New York, v. 41, n.3, p.123-127, 2012.

COSTA, M. S.; PEREIRA, M. J. B.; OLIVEIRA, S. S.; SOUSA-JUNIOR, P. T.; DALL'OGLIO, E. L.; ALVES, T. C. Anonáceas provocam mortalidade em lavras de Aedes aegypti (Linnaeus, 1762) (Diptera: Culicidae). Revista Brasileira de Biociências, Porto Alegre, v. 11, p.184-190, 2013.

CRUZ, P. E. O. Estudo fitoquímico e investigação das atividades antioxidante, antimicrobiana $\mathrm{e}$ larvicida das cascas de Annona salzmannii A. DC. (Annonaceae). 2011. 136 f. Dissertação (Mestrado em Química) - Universidade Federal de Sergipe, Aracajú, 2011.

CRUZ-ESTRADA, A.; GAMBOA-ÂNGULO. M.; BORGES-ARGÁEZ, R.; RUIZ-SÁNCHEZ, E. Insecticidal effects of plant extracts on immature whitefly Bemisia tabaci Genn. (Hemiptera: Aleyroideae). Electronic Journal of Biotechnology, Valparaíso, v. 16, n.1, 2013.

CZEPAK, C.; ALBERNAZ, K. C.; VIVAN, L. M.; GUIMARÃES, H. O.; CARVALHAIS, T. Primeiro registro de ocorrência de Helicoverpa armigera (Hübner) (Lepidoptera: Noctuidae) no Brasil. Pesquisa Agropecuária Tropical, Goiânia, v. 43, n.1, p.110-113, 2013.

DADANG, E. D. F.; PRIJONO, D. Effectiveness of two botanical insecticide formulations to two major cabbage insect pests on field application. International Society for Southeast Asian Agricultural Sciences, Parañaque, v. 15, n.1, p.42-51, 2009.
D’AMATO, C.; TORRES, J. P. M.; MALM, O. DDT (dicloro difenil tricloroetano): toxicidade e contaminação ambiental - uma revisão. Química Nova, São Paulo, v. 25, n. 6a, p. 995-1002, 2002.

DANIEL, B.; INNOCENT, E.; MBWAMBO, Z. H.; MUSHARRAF, S. G. Comparison of mosquito larvicidal activity of Annona squamosa leaves growing in different eco-zones in Tanzania. International Journal of Pharma and Bio Sciences, Guntur, v. 2, n.4, p. 566-572, 2011.

DIAS, C.N.; MORAES, D. F. C. Essential oils and their compounds as Aedes aegypti L. (Diptera: Culicidae) larvicides: review. Parasitology Research, Heidelberg, v. 112, n. 11, p. 1-28, 2013.

DILL, E. M.; PEREIRA, M. J. B; COSTA, M. S. Efeito residual do extrato de Annona coriacea sobre Aedes aegypti. Arquivos do Instituto Biológico, São Paulo, v. 79, p. 595-601-601, 2012.

EPINO. P. B.; CHANG, F.; Insecticidal activity of Annona squamosa L. seed extracts against the Mediterranean fruit fly, Ceratitis capitata (Wiedemann) (Diptera: Tephritidae). Philippine Entomologist, Filipinas, v. 9, p. 228-23, 1993.

EWETE, F. K.; ARNASON, J. T.; LARSON, J.; PHILOGÈNE, B. J. R. Biological activities of extracts from traditionally used Nigerian plants against the European corn borer, Ostrinia nubilalis. Entomologia Experimentalis et Applicata, Dordrecht, v. 80, n.3, 531-537. 1996.

FEITOSA, E. M. A.; ARRIAGA, Â. M. C.; SANTIAGO, G. M. P.; LEMOS, T. L. G.; OLIVEIRA, M. C. F.; VASCONCELOS, J.N.; LIMA, J. Q.; MALCHER, G. T.; NASCIMENTO, R. F.; BRAZFILHO, R. Chemical composition and larvicidal activity of Rollinia leptopetala (Annonaceae). Journal of the Brazilian Chemical Society, Campinas, v. 20, n.2, p.375-378, 2009.

FONTANA, J.; LANCAS, F.; PASOS, M.; CAPPELARO, E.; VILLEGAS, J.; BARON, M.; NOSEDA, M.; POMIIIO, M.; VITALE, A.; WEBBER, A.; MAUL, A.; PERES, W. Y FOERSTER, L. Selective Polarity- and adsorptionguided extraction/purification of Annona sp. polar acetogenins and biological assay against agricultural pests. Applied Biochemistry and Biotechnology, Riverside, v. 70, p.67-7, 1998. 
GALLO, D.; NAKANO, O.; SILVEIRA NETO, S.; CARVALHO, R.P.L.; BAPTISTA, G.C.; BERTI FILHO, E.; PARRA, J.R.P.; ZUCCHI, R.A.; ALVES, S.B.; VENDRAMIN, J.D. ; MARCHINI, L.C.; LOPES, J.R.S.; OMOTO, C. Entomologia agrícola. Piracicaba: FEALQ, 2002. 920p.

GONZÁLEZ-ESQUINCA, A. R.; CAZÁRES, L. M. L.; GUZMÁN, M. A. S.; CHACÓN C. I. D. C.; HERNÁNDEZ, G. L.; BRECEDA, S. F.; GERARDO, P.M. In vitro larvicidal evaluation of Annona muricata L., A. diversifolia Saff. and A. lutescens Saff. extracts against Anastrepha ludens Larvae (Diptera, Tephritidae) Interciencia, Venezuela, v. 37, n. 4, p. 284-289, 2012.

GRAINGE, M.; AHMED, S.; MITCHELL, W. C.; HYLIN, J. W. Plant species reportedly possessing pest-control properties - A database. Honolulu: Resource Systems Institute, East-West Centre, 1984. 240p.

GUADAÑO, A.; GUTIÉRREZ, C.; DE LA PEÑA, E.; CORTÉS, D. Y GONZÁLEZ, A. Insecticidal and mutagenic evaluation of two annonaceous acetogenins. Journal of Natural Products, Columbus, v. 63, p.773-776, 2000.

GUARIDO, M. M. Atividade inseticida de extratos de Annona foetida Mart. (Annonaceae) sobre imaturos de Aedes aegypti (Linnaeus, 1762) (Diptera: Culicidae). 69f. 2009. Dissertação (Mestrado Ciências Biológicas, Entomologia) Universidade Federal do Paraná, Curitiba, 2009.

HERNANDEZ, C. R.; VENDRAMIN, J. D. Toxicidad de extractos acuosos de Meliaceae em Spodoptera frugiperda (Lepidoptera: Noctuidae). Manejo Integrado de Plagas, San José, v. 42, p. 14- 22, 1996.

HEUSDEN, E. C. H. Flowers of Annonaceae: morphology, classification and evolution. Blumea Netherland, v. 7, p.1-218, 1992. Supplement .

HINCAPIÉ-LLANOS, C. A., ARANGO, D. L.; GIRALDO, M. C. Actividad insecticida de extractos de semilla de Annona muricata (Anonaceae) sobre Sitophilus zeamais (Coleoptera: Curculionidae). Revista Colombiana del Entomología, Bogotá, v. 34, n.1, p.76-82. 2008.
IMENES, S. D. L.; IDE, S. Principais grupos de insetos pragas em plantas de interesse econômico. O Biológico, São Paulo, v. 64, n.2, p.235-238, 2002.

ISMAN, M.B. Neem and other botanical insecticides: barriers to commercialization. Phytoparasitica, Berlin, v. 25, n. 4, p. 339-344, 1997.

JANMAAT, A. F.; MYERS, J. Rapid evolution and the cost of resistance to Bacillus thuringiensis in greenhouse populations of cabbage loopers, Trichoplusia ni. Proceedings of the Royal Society of London, London, v. 270, p.2263-2270, 2003.

JOST, D. J.; PITRE, H. N. Soybean looper and cabbage looper (Lepidoptera: Noctuidae) populations in cotton and soybean cropping systems in Mississippi. Journal of Entomological Science, Tifton, v. 37, p.227-235, 2002.

KABIR, K. E. Larvicidal effect of an alkaloidal fraction of Artabotrys odoratissimus (Annonaceae) bark against the filarial mosquito Culex quinquefasciatus (Diptera: Culicidae). International Journal of Tropical Insect Science, Wallingford, v. 30, n. 3, p.167-169, 2010.

KAMARAJ, C.; BAGAVAN, A.; ELANGO, G.; ZAHIR, A. A.; RAJAKUMAR, G.; MARIMUTHU, S.; SANTHOSHKUMAR. T.; RAHUMAN, A. A. Larvicidal activity of medicinal plant extracts against Anopheles subpictus \& Culex tritaeniorhynchus. Indian Journal of Medical Research, New Delhi, v. 134, p.101-106. 2011.

KARUNARATNE, M. M. S. C.; ARUKWATTA, A. P. K. M C. P. Efficacy of three plant species on the mortality and food consumption of Epilachna vigintioctopunctata. Vidyodaya Journal of Science, Nugegoda, v. 14, p.167-176, 2009.

KAWAZU, K.; ALCANTARA, J.; KOBAYASHI, A. Isolation and structure of neoannonin, a novel insecticidal compound from the seeds of Annona squamosa. Agricultural and Biological Chemistry, Tokyo, v. 53, p.2719-2722, 1989.

KEMPRAJ, V.; BHAT, S. K. Acute and reproductive toxicity of Annona squamosa to Aedes albopictus. Pesticide Biochemistry and Physiology, Amsterdam, v. 100, p.82-86, 2011. 
KESETYANINGSIH, T. W. Efficacy of Annona squamosa leaf extract as an insecticide against Cockroach (Periplaneta americana). In: INTERNATIONAL CONFERENCE: RESEARCH AND APPLICATION ON TRADITIONAL COMPLEMENTARY AND ALTERNATIVE MEDICINE IN HEALTH CARE, 2012. Surakarta. p.152-156, 2012.

KHALEQUZZEMAN, M.; SULTANA, S. Insecticidal activity of Annona squamosa L seed extracts against the red flour beetle, Tribolium castaneum (Herbst.). Journal of Bio-Science, Bangladesh, v. 14, p.107-112, 2006.

KOTKAR, H. M.; MENDKI, P. S.; SADAN, S. V. G. S.; JHA, S. R.; UPASANI, S. M.; MAHESHWARI, V. L. Antimicrobial and pesticidal activity of partially purified flavonoids of Annona squamosa. Pest Management Science, Malden, v. 58, n.1, p. 33-37, 2002.

KRINSKI, D.; MASSAROLI, A. Nymphicidal effect of Annona mucosa and A. crassiflora extracts (Annonaceae) against Tibraca limbativentris (Pentatomidae). In: INTERNATIONAL CONGRESS \& BRAZILIAN MEETING ABOUT ANNONACEAE: FROM GENE TO EXPORTATION, 5., 2013, Botucatu. Proceedings... Botucatu: Universidade Estaudal, Instituto de Biociências, 2013. p. 180-183.

LEATEMIA, J.; ISMAN, M. Efficacy of crude seed extracts of Annona squamosa against diamondback moth, Plutella xylostella L. in the greenhouse. International Journal of Pest Management, London, v. 50, p.129-133, 2004.

LEWIS, M. A.; ARNASON, J. T.; PHILOGENE, B. J. R.; RUPPRECHT, J. K.; MCLAUGHLIN, J. L. Inhibition of Respiration at Site I by Asimicin, an Insecticidal Acetogenin of the Pawpaw, Asimina triloba (Annonaceae). Pesticide Biochemistry and Physiology, Amsterdam, v. 45, n.1, p.15-23, 1993.

LOURENÇÃO, A. L.; RECO, P. C.; BRAGA, N. R.; VALLE, G. E. DO; PINHEIRO, J. B. Produtividade de genótipos de soja sob infestação da lagarta-da-soja e de percevejos. Neotropical Entomology, New York, v. 39, p. 275-281, 2010
LUCAS, P. W.; TURNER, I. M.; DOMINY, N. J.; YAMASHITA, N. Mechanical defences to herbivory. Annals of Botany, Oxford, v. 86, p.913 920, 2000.

LÜMMEN, P. Complex I inhibitors as insecticides and acaricides. Biochimica et Biophysica Acta, Amsterdam, v. 1364, n.2, p.287-296, 1998.

MAGADULA, J. J.; INNOCENT, E.; OTIENO, J. N. Mosquito larvicidal and cytotoxic activities of 3 Annona species and isolation of active principles. Journal of Medicinal Plants Research, Lagos, v. 3, n.9, p. 674-680, 2009.

MAGALHÃES, F. B.; SILVA, V. P.; PEREIRA, M. J. B.; SOUZA, P. T.; DALL'OGLIO, E. L. Efeito do extrato hexânico de Annona mucosa Jacq. (Annonaceae) na mortalidade de ninfas do percevejo Euschistus heros F. (Heteroptera: Pentatomidae). In: CICLO DE ESTUDOS EM BIOLOGIA DE TANGARÁ DA SERRA, 2., CICLO NACIONAL DE ESTUDOS DE BIOLOGIA, 1., 2011. Tangará da Serra. Anais..

MAIRESSE, L. A. S. Avaliação da bioatividade de extratos de espécies vegetais, enquanto excipientes de aleloquímicos. 2005. 329 f. Tese (Doutorado em Agronomia) - Universidade Federal de Santa Maria, Santa Maria, 2005.

MASSAROLI, A.; PEREIRA, M. J. B.; FOERSTER, L.A. Annona mucosa como fitoinseticida para o controle de Chrysodeixis includens (Lepidoptera: Noctuidae). In: SIMPÓSIO DE CONTROLE BIOLÓGICO (SICONBIOL), 13., 2013, Bonito. Anais... Brasília: Embrapa, 2013. CD-ROM.

MIKAMI, A, Y. Mortalidade do percevejo Euschistus heros (F.) (Hemiptera:Pentatomidae) provocada por extratos de sementes de Tephrosia sp. (Fabaceae) e de anonáceas. 2011. 61f. Tese (Doutorado em Agronomia) - Universidade Estadual de Londrina, Londrina, 2011.

MIKOLAJCZAK, K. J.; MADRIGAL, R. V.; RUPPRECHT, J. K.; HUI, Y. -H. ; LIU, Y. M.; SMITH, D. L.; MCLAUGHLIN, J.L. Sylvaticin: A new cytotoxic and insecticidal acetogenin from Rollinia sylvatica (Annonaceae). Experientia, Basel, v. 46, n.3, p.324-327, 1990. 
MIKOLAJCZAK, K. L.; McLAUGHLIN, J. L.; RUPPRECHT, J. K. Control of pests with annonaceous acetogenins. Washington: Secretary of Agriculture, 1988. Patent number: 4.721.727, Jan. 26, 1988

MONZON, R. B.; ALVIOR J. P.; LUCZON L. L. C.; MORALES A. S.; MUTUC F. E. S. Larvicidal potential of five Philippine plants against Aedes aegypti (Linnaeus) and Culex quinquefasciatus (Say). Southeast Asian Journal of Tropical Medicine and Public Health, Bangkok, v. 25, n.4, p. 755759, 1994.

MORAES, J. M.; PEREIRA, M. J. B.; COSTA, M. S.; GALBIATI, C.; LOUREIRO. Avaliação da atividade de Annona coriacea (Annonaceae) sobre pupas e adultos de Aedes aegypti (Diptera:Culicidae) em laboratório. Revista de Agricultura, Piracicaba, v. 86, p. 115-121, 2011.

MORALES C. A.; GONZÁLEZ, R.; ARAGÓN, R.. Evaluación de la actividad larvicida de extractos polares y no polares de acetogeninas de Annona muricata sobre larvas de Aedes aegypti y Anopheles albimanus (Diptera: Culicidae). Revista Colombiana de Entomología, Bogotá, v. 30, n.2, p.187-192, 2004.

MORAL-GARCIA, F. J. Analysis of the spatiotemporal distribution of Helicoverpa armigera (Hübner) in a tomato field using a stochastic approach. Biosystems Engineering, London, v. 93, n.3, p. 253-259, 2006.

MURTY, U. S.; SRIRAM, K.; KAISER, J. Effect of leaf extract of Polyalthia longifolia (Family: Annonaceae) on mosquito larvae and pupae of Culex quinquefasciatus (Diptera: Culicidae) of different habitats. International Pest Control, London, v. 39, n.2, p.52-53, 1997.

NAVARRO-SILVA, M. A.; MARQUES, F. A.; DUQUE, J. E. L. Review of semiochemicals that mediate the oviposition of mosquitoes: a possible sustainable tool for the control and monitoring of Culicidae. Revista Brasileira de Entomologia, Curitiba, v. 53, n.1, p.1-6, 2009.

NGAMO-TINKEU, L. S.; GOUDOUM, A.; NGASSOUM, M. B.; MAPONGMETSEM, P. M.; KOUNINKI, H.; HANCE, T. Persistence of the insecticidal activity of five essential oils on the maize weevil Sitophilus zeamais (Motsch.) (Coleoptera:
Curculionidae). Communications in Agricultural and Applied Biological Sciences, Gent, v. 69, n.3, p.145-147, 2004.

NYANDORO, S. S.; JOSEPH, C. C.; NKUNYA, M. H. H.; HOSEA, K. M. M. New antimicrobial, mosquito larvicidal and other metabolites from two Artabotrys species. Natural Product Research: Formerly Natural Product Letters, London, v. 27, n. 16, p.1450-1458, 2013.

ODALO, J. O.; OMOLO, M. O.; MALEBO, H.; ANGIRA, J.; NJERU, P. M.; NDIEGE, I. O.; HASSANALI, A. Repellency of essential oils of some plants from the Kenyan coast against Anopheles gambiae. Acta Tropica, New York, v. 95, n.3, p.210-218, 2005.

OKONKWO, E. U.; OKOYE, W. I. Insecticidal activity of Dennettia tripetala Baker f. and Piper guineense Schum. and Thonn. against Dermestes maculatus Degeer (Coleoptera: Dermestidae) and Necrobia rufipes Degeer (Coleoptera: Cleridae) on dried fish. Nigerian Journal of Entomology, Ibadan, v. 18, p.109-117, 2001.

OLIVEIRA, A. C.; PEREIRA, M. J. B. Efeito antialimentar do extrato metanólico de Annona crassiflora Mart. sobre o percevejo marrom Euschistus heros (Fabr. 1798)(Heteroptera:Pentatomidae). Revista Brasileira de Agroecologia, Porto Alegre, v. 4, n.2, p.2633-2636, 2009.

OLIVEIRA, S. S.; PEREIRA, M. J. B.; COSTA, M. S.; JUNIOR, P. T. S.; DALL'OGLIO, E. L. Bioatividade do extrato hexânico bruto de Annona crassiflora no controle do vetor da dengue aedes aegypti 1. (diptera: culicidae). In: CONGRESSO DE INICIAÇÃO CIENTÍFICA, 3., 2010, Cáceres. Anais... Cáceres: Pró-Reitoria de Pesquisa e PósGraduação, 2010. v.6, CD-ROM.

OMENA, M. C.; NAVARO, D. M. A. F.; PAULA, J. E.; LUNA, J. S.; LIMA, M. R. F.; SANT’ANA, A. E. G. Larvicidal activities against Aedes aegypti of some Brazilian medicinal plants. Bioresource Technology, New York, v. 98, n.13, p.2549-2556, 2007.

OWUSO, D. O. Effect of crude ethanolic leaf extract of soursop Annona muricata L.) on eggplant shoot and fruit borer (Leucinodes orbonalis guen.). 2012. 65f. Tese (Master of Science) - University of Cape Coast, Ghana, 2012. 
PARRA-HENAO, G.; GARCÍA, C.; COTES, J. Actividad insecticida de extractos vegetales sobre Aedes aegypti (Diptera: Culicidae) vector del dengue en Colombia. Revista CES Medicina, Medellín, v. 21, p.47-54,2007a.

PARRA-HENAO, G.; GARCÍA, C.; COTES, J. Actividad insecticida de extractos vegetales sobre Rhodnius polixus y Rhodnius pallescens (Hemiptera: Reduviidae). Boletín de Malariología y Salud Ambiental, Maracay, v. 47, p.125-137, 2007b.

PÉREZ-PACHECO, R.; RODRÍGUEZ, C.; LARA, J.; MONTES, R.; VALVERDE, G. Toxicidad de aceites, esencias y extractos vegetales en larvas de mosquito Culex quinquefasciatus Say (Diptera: Culicidae). Acta Zoológica Mexicana, Veracruz, v. 20, p.141-152, 2004.

POGUE G. M. A world revision of the genus Spodoptera Guenée (Lepidoptera: Noctuidae). Memoirs of the American Entomological Society, Philadelphia, v. 43, n.28, p.117-124, 2002.

POGUE, M. G. A new synonym of Helicoverpa zea (Boddie) and differentiation of adult males of $H$. zea and $H$. armigera (Hübner) (Lepidoptera: Noctuidae: Heliothinae). Annals of the Entomological Society of America, College Park, v. 97, n.6, p.1222-1226, 2004.

PRATES, H. T.; VIANA, P. A.; PIMENTA, L. P. S.; BOAVENTURA, M. A. D. Insecticide action of ethanolic extract from Annona crassiflora seeds against Spodoptera frugiperda. In: INTERNATIONAL CONGRESS OF ENTOMOLOGY, 21., 2000, Foz do Iguassu. Abstract Book... Londrina: Embrapa Soja, 2000. v. 1, p. 1035.

PRATIBHA V. D.; HOOLI, A. A.; HOLIHOSUR, S. N. Bioefficacy of cold ethyl alcohol extract of Annona squamosa against Spodoptera litura Fabricius. Journal of Biopesticides, Tamilnadu, v. 3, n.1, p.271-274, 2010.

QADRI, S. S. H.; RAO.; B. B. Effect of combining some indigenous plant seed extracts against household insects. Pesticides, Bombay, v. 11, p.2123, 1977.

RAO, N. S.; SHARMA, K.; SHARMA, R. K. Antifeedant and growth inhibitory effects of seed extracts of custard apple, Annona squamosa against Khapra Beetle, Trogoderma granarium. Journal of Agricultural Technology, Bangkok, v. 1, n.1, p.43-54, 2005.
RATNAYAKE, S.; RUPPRECHT, J. K.; POTTER, W. M.; McLAUGHLIN, J. L. Evaluation of the pawpaw tree, Asimina triloba (Annonaceae), as a commercial source of the pesticidal annonaceous acetogenins. In: JANICK, J.; SIMON, J.E. (Ed.) New crops. New York: Wiley, 1993. p. 644-648.

REDDY K. D.; BHAGAWAN, C. N.; SUKUMAR, K.. Depletion of amino acids and protein in the nymphal haemolymph of the red cotton bug, Dysdercus koenigii (Fab.) (Hemiptera: Pyrrhocoridae) treated with Annona squamosa extract. Phytophaga, Calcutta, v. 5, p.45-50, 1993.

RIBEIRO, L. P.; VENDRAMIM, J. D.; BICALHO, K. U.; ANDRADE, M. S.; FERNANDES, J. B.; MORAL, R. A.; DEMÉTRIO, C. G. B. Annona mucosa Jacq. (Annonaceae): A promising source of bioactive compounds against Sitophilus zeamais Mots. (Coleoptera: Curculionidae). Journal of Stored Products Research, Exeter, v. 55, p. 6-14, 2013.

ROARK, R. C. Some promising insecticidal plants. Economic Botany, New York, v.1, p.437-445. 1947.

ROBLEDO-REYES，P.; GONZÁLEZ，R.; JARAMILLO, G.; RESTREPO, J. Evaluación de la toxicidad de acetogeninas annonáceas sobre ninfas de Periplaneta americana L. (Dyctioptera: Blattidae). Boletín del Museo de Entomología de la Universidad del Valle, Meléndez, v. 9, n.1, p.54-61, 2008

RODRIGUES,A.M. S.; PAULA, J.E.; DEGALLIER, N.; MOLEZ, J. F.; ESPINDOLA, L. S. Larvicidal activity of some Cerrado plants extracts against Aedes aegypti. Journal of the American Mosquito Control Association, Mount Laurel, v. 22, n.2, 314$317,2006$.

ROJANO, B.; GAVIRIA, C.; SÁEZ, J.; YEPES, F.; MUÑOZ, F. Y.; OSSA, F. Berenjenol aislado de Oxandra cf. xylopioides (Annonaceae) como insecticida. Vitae, Medellín, v. 14, p.95-100, 2007.

SAITO, M. L.; POTT, A.; FERRAZ, J. M. G.; NASCIMENTO, R. dos.S. Avaliação de plantas com atividade deterrente alimentar em Spodoptera frugiperda (J. E. Smith) e Anticarsia gemmatalis Hubner. Pesticidas: Revista de Ecotoxicologia e Meio Ambiente, Curitiba, v. 14, p.1-10, 2004. 
SALAZAR, E. C. Inseticidas e acaricidas. Pelotas: UFPelotas, 1997. 646p.

SAUQUET, H.; DOYLE, J. A.; SCHARASCHKIN, T.; BORSCH, T.; HILU, K.; CHATROU, L. W.; LE THOMAS, A. Phylogenetic analysis of Magnoliales and Myristicaceae based on multiple data sets: implications for character evolution. Botanical Journal of the Linnean Society, London, v. 142, p.125-186, 2003.

SAXENA, R. C.; HARSHAN, V.; SAXENA, A.; SUKUMARAN, P.; SHARMA, M. C.; KUMAR, M. L. Larvicidal and chemosterilant activity of Annona squamosa alkaloids against Anopheles stephensi. Journal of the American Mosquito Control Association, Mount Laurel, v. 9, n.1, p.84-87, 1993.

SCHLÜTER, M. A. Avaliação do potencial de extratos vegetais no controle de Anticarsia gemmatalis Hübner em campo. 2006. $77 \mathrm{f}$. Dissertação (Mestrado) - Universidade Federal de Santa Maria, Santa Maria, 2006.

SCHMEDA-HIRSCHMANN, G.; ARIAS, A.R. A Screening Method for Natural Products on Triatomine Bugs. Phytotherapy Research, New York, v. 6, p.68-73, 1992.

SEFFRIN, R. C.; IKKEI, S.; YASMIN A.; MURRAY B. I. Effects of crude seed extracts of Annona atemoya and Annona squamosa L. against the cabbage looper, Trichoplusia $n i$ in the laboratory and greenhouse. Crop Protection, Amsterdam, v. 29, n.1, p. 20-24, 2010.

SHARMA, P. P.; PARDESHI, A. B.; VIJIGIRI, D. Bioactivity of some medicinal plant extracts against Musca domestica L. Journal of Ecobiotechnology, Humnabad, v. 3, n.9, p.14-16, 2011.

SILVA, A.P.T.; PEREIRA, M. J. B.; BENTO, L.F. Extrato metanólico da semente de araticum (Annona coriacea) (MART.) sobre a mortalidade da traça-dotomateiro (Tuta absoluta). Revista Brasileira de Agroecologia, Cruz Alta, v. 2, n.2, p. 1150-1153, 2007.

SILVA, V. P.; PEREIRA, M. J. B.; TURCHEN, L. M. Efeito de extratos vegetais no controle de Euschistus heros (F.) (Hemiptera: Pentatomidae) em lavoura de soja na região sudoeste do Estado de Mato Grosso. Revista de Agricultura, Piracicaba, v. 88, p. 185190, 2013.
SILVA, L. B.; SILVA, J. C.; PAVAN, B. E.; PEREIRA, F. F.; MAGGIONI, K.; ANDRADE, L. H.; CANDIDO, A. C. S.; PERES, M. T. L. P. Insecticide irritability of plant extracts against Sitophilus zeamais. African Journal of Agricultural Research, Nairobi, v. 8, n.11, p. 978-983, 2013.

SINCHAISRI, N.; ROONGSOOK, D.; CHUNGSAMARNYART, N. Insecticidal activity of plant crude extracts on diamondback moth larvae. Kasetsart Journal: Natural Science, Bangkok, v. 25, n.5, p.106-110, 1991. Supplement

SOUZA, E. M.; CORDEIRO, J. R.; PEREIRA, M. J. B. Avaliação da atividade inseticida dos diferentes extratos das sementes de Annona coriacea sobre Dichelops melacanthus (Dallas, 1851). In: Resumos do V CBA - Manejo de Agroecossistemas Sustentáveis. Revista Brasileira de Agroecologia, Cruz Alta, v.2, n.2, p.1107-1110, 2007.

SREELETHA, C.; GEETHA, P. R. Pesticidal effects of Annona squamosa L. on male Oryctes rhinoceros Linn. (Coleoptera: Scarabaeidae) in relation to reproduction. Current Biotica, New Delhi, v. 6, n.1, p.8-21, 2012.

TOLOSA, D.; ÁLVAREZ-COLOM, O.; BARDÓN, A.; NESKE. A. Insecticidal effects of acetogenins from Rollinia occidentalis seed extract. Natural Product Communications, Westerville, v. 7, n.12, p.1645-1646, 2012.

TONG, H.; SU, Q.; ZHOU, X.; BAI, L. Field resistance of Spodoptera litura (Lepidoptera: Noctuidae) to organophosphates, pyrethroids, carbamates and four newer chemistry insecticides in Hunan, China. Journal of Pest Science, Heidelberg, v. 86, n.3, p.599-609, 2013.

TRINDADE, R. C. P.; LUNA, J. S.; LIMA, M. R. F.; SILVA, P. P.; SANT'ANA, A. E. G. Larvicidal activity and seasonal variation of Annona muricata (Annonaceae) extract on Plutella xylostella (Lepidoptera: Plutellidae). Revista Colombiana de Entomología, Bogotá, v. 37, n.2, p.223-227, 2011.

UKEH, D. A.; ADIE, E. B.; UKEH, J. A. Insecticidal and repellent activities of pepper fruit, Dennettia tripetala (G. Baker) against the cowpea beetle, Callosobruchus maculatus (F.). Biopesticides International, Ghaziabad, v. 7, n.1, p.15-23, 2011. 
UKEH, D. A.; ARONG, G. A.; OGBAN, E. I. Toxicity and oviposition deterrence of Piper guineense (Piperaceae) and Monodora myristica (Annonaceae) against Sitophilus zeamais (Motsch.) on stored maize. Journal of Entomology, London, v. 5, n.4, p.295-299, 2008.

UKEH, D. A.; OKU, E. E.; UDO, I. A.; NTA, A. I.; UKEH, J. A. Insecticidal effect of fruit extracts from Xylopia aethiopica and Dennettia tripetala (Annonaceae) against Sitophilus oryzae (Coleoptera: Curculionidae). Chilean Journal of Agricultural Research, Santiago, v. 72, n.2, p.195-200, 2012.

UMEOTOK, S. B. A.; ISAH, M. D.; UKEH, J. A.; UDO, I. A. Evaluation of the insecticidal and deterrence properties of pepper fruit, Dennettia tripetala (G. Baker) and Ginger Zingiber officinale Roscoe against maize weevil Sitophilus zeamais (Motsch.). Journal of Biology, Agriculture and Healthcare, Nigeria, v. 3, n.6, p.76-82, 2013.
VIEGAS-JUNIOR, C. Terpenos com atividade inseticida: uma alternativa para o controle químico de insetos. Química Nova, São Paulo, v. 26, n.3, p.390-400, 2003.

ZAFRA-POLO, M. C.; GONZÁLES, M. C.; ESTORNELL, E.; SAHPAZ, S.; CORTÉS, D. Acetogenins from Annonaceae, inhibitor of mitocondrial complex I. Phytochemistry, Oxford, v.42, p.253-271, 1996.

ZARIDAH, M. Z.; NOR AZAH, M. A.; ROHANI, A. Mosquitocidal activities of Malaysian plants. Journal of Tropical Forest Science, Kuala Lumpur, v. 18, n.1, p. 74-80, 2006. 